\title{
Urgences
}

\section{Les lieux sauvages}

\section{Domingo Cisneros}

Numéro 17-18, octobre 1987

L'esprit des lieux

URI : https://id.erudit.org/iderudit/025417ar

DOI : https://doi.org/10.7202/025417ar

Aller au sommaire du numéro

Éditeur(s)

Urgences

ISSN

0226-9554 (imprimé)

1927-3924 (numérique)

Découvrir la revue

Citer ce document

Cisneros, D. (1987). Les lieux sauvages. Urgences, (17-18), 19-27.

https://doi.org/10.7202/025417ar

Ce document est protégé par la loi sur le droit d'auteur. L'utilisation des services d'Érudit (y compris la reproduction) est assujettie à sa politique d'utilisation que vous pouvez consulter en ligne.

https://apropos.erudit.org/fr/usagers/politique-dutilisation/
Cet article est diffusé et préservé par Érudit.

Érudit est un consortium interuniversitaire sans but lucratif composé de l’Université de Montréal, l'Université Laval et l'Université du Québec à Montréal. Il a pour mission la promotion et la valorisation de la recherche. https://www.erudit.org/fr/ 


\section{Domingo Cisneros \\ LES LIEUX SAUVAGES}

Il y a vingt ans, je ne savais pas que certains lieux avaient le pouvoir d'ensorceler. Pourtant, depuis lors je suis ensorcelé par l'un d'eux. Ce lieu, le Québec, est aussi coupable du fait que je me suis trouvé en tant qu'homme et artiste.

Je demeure dans le nord des Laurentides, dans la région de la Vallée de la Rouge, aux alentours d'un petit village qui s'appelle La Macaza. J'y suis arrivé en 1974 pour enseigner l'art autochtone au Collège Manitou, un centre éducatif pour Inuit, Indiens et Métis.

La Macaza ce fut l'amour à première vue. Ou même avant la première vue. L'idylle a commencé par le mot, le simple nom: La Macaza. En espagnol, c'est "chez-nous" et, en même temps, "ma chasse". Il y avait de la magie là. Je venais de passer presqu'un an aux lles Fidjii, et maintenant j'avais l'occasion d'aller vivre en forêt. En plus, j'allais participer à une expérience collective: la lutte pour la renaissance de l'esprit autochtone.

Le collège est situé sur une ancienne base de missiles nucléaires. Vite devenue surannée, la base a été abandonnée par les forces américaines et canadiennes. Le Ministère de la Défense, n'y ayant pas trouvé de nouvelle vocation militaire, l'a louée à l'Association des Indiens du Québec pour un dollar par année. Ainsi est né ce lieu, ce rêve de courte durée.

C'est étrange comment les lieux changent d'esprit. Tout d'abord un lieu de paranoīa nucléaire, ensuite un éléphant blanc perdu dans le bois, puis un centre de reprise d'identité et de renaissance, pour enfin devenir aujourd'hui, mauvaise plaisanterie, un pénitencier fédéral. Je sais que les divinités boréales autour de cette place sont aussi perplexes que moi. Tout ce va-et-vient d'âmes en peines, d'esprits proscrits. Mais à l'époque, il y a treize ans, nous remontions aux sources. Tout était à faire. La tâche était immense, mais l'enthousiasme était encore plus grand.

L'artiste, pour naître comme tel, a tué son moi chamanique et a filé du paradis. Nous, au contraire, devions retourner à l'état chamanique par la porte des esprits du bois pour briser le complot millénaire. Nous devions les connaître, communiquer avec eux, et parfois apaiser leurs colères ou leurs extravagances. La renaissance artistique des nations indigènes en dépendait beaucoup. Bien s'entendre avec 
la flore et la faune, les esprits intermédiaires, était une condition fondamentale de survie. Notre chemin nous conduisait vers le cuivre, la pierre et l'os, dans un voyage contre le courant du temps.

Le lieu était idéal. Nous avons commencé l'aventure armés de haches, de couteaux et de poinçons. Une grande partie du temps nous l'avons passé dans le bois à cueillir des matériaux, à chercher l'énergie par nos invocations, à scruter le corps de la Mère Terre, à converser et à rêver, à chercher des visions, à confronter des esprits. Nous avons invité des "medicine men" et des aînés, pour qu'ils nous transmettent leur sagesse. Il y a eu des échanges avec des réserves autochtones. Nous avons organisé une conférence des artistes et artisans de la forêt de l'Est du Canada. Nous avons suivi des cours de danse, de poésie, de théâtre, de littérature. Nous avons organisé des coopératives et des expositions, dressé des programmes d'enseignement, publié des écrits. L'esprit autochtone fleurissait. La Macaza s'est transformée en centre de liaison, en une espèce de Mecque autochtone. Parmi les étudiants il y avait des Inuit du Grand Nord Québécois; des Iroquois de la Ligue des Six Nations; des Cris et des Algonquins de l'Abitibi et de la Baie James; des Micmacs et des Amalécites des Maritimes; des Montagnais et des Naskapis de la Côte Nord; des Attikameks de la Mauricie; des Abénakis et des Hurons de la Laurentie; des Ojibwés de l'Ontario; des Cris et des Pieds-Noirs des Prairies.

Non seulement l'esprit du lieu s'est-il transformé en réalité, mais sa renommée s'est rendue à des endroits toujours plus lointains. Mais cette renommée, gagnée en trois ans et demi, causait un malaise au Ministère des Affaires indiennes, qui a décidé de fermer le Collège. La même histoire se répétait encore une fois. Un autre rêve brisé, un autre esprit blessé.

En décembre 1976, en plein hiver, les dernières familles ont été délogées. Une grande partie du travail réalisé en Arts et communications a été tout simplement enterré, à l'aide d'un bulldozer. Des centaines d'heures d'enregistrement vidéo, des milliers de photos et de négatifs, des oeuvres d'art, des matériaux de travail et de recherche, et même des outils sont disparus ainsi. Néanmoins, tout n'a pas été détruit. Quelques-uns de nos jeunes se sont sauvés de l'alcoolisme, de la violence, du suicide, et aujourd'hui ils continuent à propager l'esprit de ce lieu, convertis en artisans, professeurs, travailleurs sociaux, chefs de bande, artistes, recherchistes, chamans, cinéastes et conseillers.

Quelques esprits s'en vont, d'autres restent. Celui du Collège 
Manitou en a laissé un fort intéressant ici. Il semble que le comté de Labelle comporte la plus grande population métisse au Québec. Pourtant, je ne veux pas laisser entendre qu'il y a eu une explosion érotique entre les compagnons du Collège Manitou et la population locale. Ce qui s'est passé c'est que la présence forte et positive de Manitou a provoqué des questions à l'égard des ancêtres. En effet, comme tant de Québécois, beaucoup de gens se sont trouvés du sang autochtone et, fiers d'être métis, ont tenu à l'authentifier, parfois avec de vieux documents paroissiaux.

Les esprits de Manitou resteront vivants, rien ne pourra les faire taire. Cependant, je suis resté dans la région, comme quelques autres professeurs et étudiants. J'ai poursuivi ma recherche dans la forêt, ce lieu qui me tenait ensorcelé, mais qui me révélait généreusement ses mystères. Et, un jour, j'ai découvert qu'en vérité je n'étais pas à La Macaza, mais dans mon enfance, dans la Sierra Madre, peut-être à Tepehuanes, où ma mère est venue au monde. Les mêmes épinettes, les mêmes ours. Je me sentais comme sij'étais le produit d'un lointain désir infantile. Ce même état confus où je croyais habiter deux endroits en même temps, ou de croire que l'un d'eux soit la cause de l'autre, m'est arrivé à Rabi, une île des Fidji. Cette fois-là, j'avais la sensation de ne pas être aux Fidji, mais plutôt quelque part au Mexique, au bord de la mer. Donc, qu'est-ce que je faisais là, qu'est-ce que je cherchais sur cette île perdue dans les mers du Sud? N'était-ce pas un lieu semblable à beaucoup d'autres le long des côtes d'Oaxaca, de Guerrero, de Tabasco ou du Yucatan? Les mêmes palmiers, plages, bananes, coquilles, mangues. Qu'est-ce qui m'avait attiré aux lles Fidji? Je savais que je trouvais d'autres nations d'autochtones avec des sagesses différentes de la mienne, des sagesses de la mer et de la jungle. En plus, je me suis rendu compte que le simple goût de l'aventure y était pour beaucoup. Car c'était un lieu lointain et reculé, à l'autre bout du monde, avec un nom qui, encore, m'a séduit.

J'ai continué à La Macaza mon labeur de loup et de corbeau, ramassant les cadavres de la forêt, les mangeant, tannant leurs peaux, momifiant, nettoyant des ossements, ramassant des écorces et des racines, faisant des expériences avec des branches et des mousses, des cornes et des sabots. Mais j'étais fâché. Les nouvelles oeuvres que je faisais étaient très différentes de celles de la période du Collège Manitou. La mort de ce dernier m'avait laissé une cicatrice. Mes liens intimes avec la nature changèrent. Pour un certain temps, j'en ai même perdu l'amour et le respect. Je l'ai traitée de traîtresse, de prostituée, d'imbécile. Au lieu de m'en approcher avec humilité, je faisais preuve d'un orgueil démesuré. Mon contact avec elle avait été celui d'un enfant, d'un saint, et maintenant je faisais mon entrée avec 
d'autres masques. Ainsi, par exemple, me moquant de la peur ancestrale provoquée par les lieux éloignés, surtout la nuit, j'y entrais, je communiquais avec ses esprits et je commençais à les défier. D'autres fois je restais là avec eux, sans rien leur dire. J'apportais parfois une bouteille de tequila, suffisamment de marijuana, quelques pilules d'acide ou de mescaline. Ah, la maudite putain, je commençais déjà à connaître ses autres visages. Je me sentais le diable du bois. J'étais fier d'attraper et de tuer, de manipuler les esprits, de défier qui que ce soit qui se mettait sur mon chemin. C'était une période amère et dure, mais d'une intense activité créatrice. Beaucoup de projets me sont venus à la tête. Un des plus persistants fut celui d'une "Attaque à la ville», dans lequel je déverserais toute ma rage contre le monde urbain, que je détestais plus que jamais. Aujourd'hui, dix ans plus tard, je dois admettre que l'idée de ce projet m'habite toujours. Pas pour donner des coups d'épée dans l'eau, mais bien pour critiquer certains aspects, certaines valeurs urbaines qui continuent à provoquer chez moi une certaine colère.

Les noms réussissent parfois à invoquer, à capter l'esprit d'un lieu. Dans ce cas, le premier contact avec l'esprit se fait au moyen d'un son qui résonne de manière spéciale, et qui trouve son écho en nous. Les noms autochtones ont souvent cette caractéristique, et, de fait, les autochtones ne donnaient pas de nom aux lieux sans y avoir d'abord observé, exploré, senti les esprits. Ils n'imposaient pas les noms, ils les découvraient.

Comme ailleurs dans les Amériques, les gens qui sont venus coloniser ce beau pays n'ont que rarement essayé de communiquer avec les esprits des lieux sauvages, qui constituaient alors le pays au complet. Il n'ont réussi qu'à confronter et à conquérir ces esprits, ou ils les ont tout simplement ignorés. lls sont venus imposer et s'imposer. Imposer des noms de saints chrétiens sur des lieux habités par des esprits paīens. Des noms de militaires et d'hommes d'état sur des lieux habités par des esprits paisibles ou anachistes. Donner des noms de grandes villes européennes aux clairières dans la grande forêt vierge. Ils ne pouvaient voir la terre qu'en tant que propriété, ne lui assigner aucune valeur sauf la valeur économique. Ils sont venus défricher, domestiquer, contrôler. Ils croyaient mettre de l'ordre dans ces lieux, ignorant que l'ordre y était déjà, et que leur intervention sabotait cet ordre. Même le mot "sauvage" était imbu d'un sens péjoratif: quelqu'un, quelque chose, quelque part n'ayant pas bénéficié des influences supérieures de la civilisation. Pire encore, on utilise ce mot comme un jugement moral négatif, une accusation de cruauté, de brutalité. 
J'ai continué à La Macaza mon labeur de loup et de corbeau, ramassant les cadavres de la forêt, les mangeant, tannant leurs peaux, momifiant, nettoyant des ossements, ramassant des écorces et des racines, faisant des expériences avec des branches et des mousses, des cornes et des sabots. Mais j'étais fâché. Les nouvelles oeuvres que je faisais étaient très différentes de celles de la période du Collège Manitou. La mort de ce dernier m'avait laissé une cicatrice. Mes liens intimes avec la nature changèrent. Pour un certain temps, j'en ai même perdu l'amour et le respect. Je l'ai traitée de traîtresse, de prostituée, d'imbécile. Au lieu de m'en approcher avec humilité, je faisais preuve d'un orgueil démesuré. Mon contact avec elle avait été celui d'un enfant, d'un saint, et maintenant je faisais mon entrée avec d'autres masques. Ainsi, par exemple, me moquant de la peur ancestrale provoquée par les lieux éloignés, surtout la nuit, j'y entrais, je communiquais avec ses esprits et je commençais à les défier. D'autres fois je restais là avec eux, sans rien leur dire. J'apportais parfois une bouteille de tequila, suffisamment de marijuana, quelques pilules d'acide de mescaline. Ah, la maudite putain, je commençais déjà à connaître ses autres visages. Je me sentais le diable du bois. J'étais fier d'attraper et de tuer, de manipuler les esprits, de défier qui que ce soit qui se mettait sur mon chemin. C'était une période amère et dure, mais d'une intense activité créatrice. Beaucoup de projets me sont venus à la tête. Un des plus persistants fut celui d'une "Attaque à la ville», dans lequel je déverserais toute ma rage contre le monde urbain, que je détestais plus que jamais. Aujourd'hui, dix ans plus tard, je dois admettre que l'idée de ce projet m'habite toujours. Pas pour donner des coups d'épée dans l'eau, mais bien pour critiquer certains aspects, certaines valeurs urbaines qui continuent à provoquer chez moi une certaine colère.

Un jour j'ai pensé que j'avais épuisé, dans le sens artistique, les possibilités que ces forêts boréales m'offraient. Mais ce n'était pas le cas. La récolte des esprits est inépuisable, comme l'est, heureusement, la soif de les décrire. Les saisons et le travail ont continué. Des printemps de sèves et d'écorces, des étés de branches et de bois, des automnes d'os et de cadavres, des hivers de création et de lecture. Voilà: pouvoir vivre chaque saison est déjà un don splendide. Surtout pour cet individu provenant d'un pays qui vante ses lieux de printemps éternel. Quant au froid, je dois admettre qu'il fut un adversaire respectable, un esprit de beaucoup de présence et de poids. Mais tout va bien entre nous maintenant. Notre relation en est une d'amitié et de respect mutuel.

À la mer, au désert, ou à la crête des montagnes, on se perd dans un paysage d'horizons lointains. Ici, dans le bois, où la frontière 
du regard est si proche, on se trouve comme enfermé avec soimême. Le voyage spirituel, donc, est nécessairement vers l'intérieur de soi. Dans ce lieu, tout est proche, immédiat. Les dieux ne sont pas faits d'astres et de couleurs, il sont de peau et d'écorce. La magie s'exerce avec des fleurs, des mousses et des animaux. Ici, il n'y a pas de permanence. La forêt est la solitude, comme la jungle. Comme le temps et les estomacs, elle dévore tout. Ce n'est pas une fin, mais plutôt le vagin, la mère, le commencement.

J'ai toujours eu des problèmes avec les lieux. Tout a commencé, j'imagine, avec mon entrée surréaliste en ce monde. Le fait sinistre d'être venu au monde dans un salon de pompes funèbres, a marqué ma vie et mon oeuvre. Je porte ce lieu toujours sur le dos, comme un corbeau à l'épaule. Avec le temps, on finit par s'habituer à tout.

À cause de ce lieu-là, la mort est toujours présente dans mon oeuvre, qui est créée à partir de cadavres de la nature, de ces pauvres esprits qui ont succombé au cycle quotidien de vie-mort. Et lorsqu'on est, comme moi, un acteur participant dans cette tragédie et non un simple spectateur, bien qu'on ne le veuille pas, on se tache de sang et d'excréments. Le travail est sale. Etriper, écorcher, désosser: le travail d'un boucher plutôt que d'un artiste. Pourtant, si on vit dans le bois, il faut le faire. Ici la vie est une tragédie continuelle, un champ de bataille. Et cela ne peut pas être autrement.

Cette Mère Terre n'a ni sentiment ni honte. Autant elle peut être belle et bénéfique, autant elle peut être macabre et traîtresse. Masques ténébreux, esprits maléfiques, ces derniers aspects sont ceux qui m'intéressent chez elle. Et par cela je ne veux pas dire que je m'abstiens de vivre d'autres choses avec elle. Non j'aime son beau corps, sa peau bénéfique et la saveur de ses fruits.

Vers la fin de l'été 1983, question de donner suite à une idée, je suis allé un temps à Durango où j'ai passé les treize premières années de ma vie. Je voulais confronter les mythes de l'enfance et entreprendre une nouvelle oeuvre dans le désert. Me trouver de nouveau avec les esprits infantiles, visiter les anciens lieux magiques, vérifier s'ils avaient changé, ou si c'était moi, ou les deux ensemble. Tout cela a été une expérience réussie.

Les traits principaux du Nord mexicain sont le manque d'eau et l'absence de culture. Ces plaines arides et poussiéreuses, ces montagnes dénudées par le vent, sont les régions les plus désolées du Mexique. Et les plus pauvres. Les touristes qui entrent au pays en 
voiture traversent le nord en toute hâte afin d'atteindre le centre, le sud et les côtes. Le Mexicain, pour sa part, abhorre le désert et, s'il l'habite, rêve d'émigrer vers des terres plus bienveillantes. Ce mépris pour le Nord mexicain remonte au temps des Aztèques, qui utilisaient le vocable péjoratif de "Chichimecas" pour désigner les tribus nomades de ces lieux, mot dont la traduction est à peu près "hommebête», "sale sauvage». Aujourd'hui encore le Mexicain du centre ou du sud pense que ceux du nord ne sont pas des citoyens à plein titre, qu'ils sont moitié Chicanos, moitié gringos. Qu'ils n'ont rien apporté à la culture nationale, sauf quelques lumières sporadiques qui ont émigré à la capitale aussitôt qu'elles pouvaient le faire pour enfin cultiver et faire fleurir leurs talents. Par contre, pour moi, ce furent ces lieux qui ont nourri mon imagination et m'ont poussé vers les chemins de la création. J'étais excité d'être de retour dans le désert, l'école d'art de mon enfance. Au lieu de me sentir angoissé à cause de l'absence de culture, je me réjouissais de l'idée qu'il n'y avait presque. rien, que tout était à faire, que le territoire était vierge, inexploré.

C'est à cette époque que j'eus ce premier contact avec un des lieux les plus fascinants que j'aie jamais trouvé: la Zone du Silence. Comme cela s'était passé avec La Macaza et les lles Fidji, je suis resté ensorcelé par la simple magie de son nom. La Zona del Silencio. Un désert magnétique où les ondes de radio ne se propagent pas, où les boussoles deviennent folles, où il y a un grand nombre de mutations chez les plantes et les animaux. Quel matériel mythique pour créer. L'ancienne Mer de Thétis aussi, pleine de fossiles des premiers habitants de la planète. Vallée de météorites, peinture rupestres, cimetières autochtones.

Le projet Zona del Silencio a pris forme pendant l'hiver 19841985 , après mille préparatifs compliqués. Nous avons campé un mois dans les ruines Apaches de Mohovano, une oasis en plein centre de la Zone. Nous étions douze artistes, représentant dix disciplines des arts, provenant de quatre pays et parlant trois langues. Chacun, selon son art, a réalisé des oeuvres tant personnelles que collectives. Dans cet endroit tout à fait isolé, nous avons joui d'une rupture totale avec le monde civilisé. Pendant quelques jours il n'y avait même pas de véhicule pour quitter, en cas d'urgence, cet espace qu'on partageait avec des scorpions, des serpents à sonnettes et des tarentules. D'une certaine façon, nous avons'risqué la vie par amour de l'art. Le projet voulait aller au-delà de la relation artistenature, du land art ou de l'art écologique. J'ai proposé qu'on appelle notre expérience «Art-aventure: une célébration des arts et du désert». 
Quand on parle du désert, on pense immédiatement aux esprits lumineux, secs, célestes, stoïques, chauds. Mais il y en a un autre, très important et peu connu, qui est né du métissage des cataclysmes terrestres et des ambitions humaines. Il est l'ennemi des pluies et des racines, mais le soleil et le vent sont ses alliés. Il fissure, crevasse, ronge, triture tout ce qu'il trouve en passant. Cet esprit est si fort qu'il est le seul parmi les esprits sauvages qui se multiplie et se répand, pendant que les autres disparaissent de jour en jour. Le visiter, le connaître, c'est voyager vers le futur de la planète.

Les différences de caractère spirituel entre un lieu sauvage et un autre domestiqué par l'être humain, sont évidentes. L'inspiration que le premier nous donne est plus vigoureuse, plus profonde et, disonsle, plus éternelle. C'est une espèce de vécu préculturel, de caractère cosmique, sacré. Le lieu sauvage est ce qui n'a pas encore été. C'est une frontière, un jusqu'ici de la culture. C'est pour cela que, dans ce genre de lieu, je n'aime pas laisser des traces qui perdurent. Je ne veux pas que mon activité artistique s'impose sur eux. En plus d'être une offense écologique, ce serait un sacrilège. L'oeuvre se laisse aux esprits du lieu, qui peuvent la réclamer, la récupérer en tous ses éléments.

Le lieu sauvage est un lieu de thérapies, de retrouvailles et de libérations. Il peut aussi être, à travers l'esprit animal, un lieu d'initiation à un autre genre de connaissance.

Le rythme de disparition de l'habitat sauvage est proportionnel à la croissance des villes. Le problème ne serait pas si grave si les dommages qu'elles causent se limitaient à leurs alentours. Mais nous savons que ce n'est pas ainsi. L'appétit vorace de ces aberrations écologiques n'est jamais satisfait. C'est une chose de vivre en ruche et de se comporter comme des abeilles. C'est une autre chose de vivre en ruche et de se comporter comme des reines. Il y a quelques années, il était impossible d'imaginer qu'un jour ces forêts boréales, si éloignées des grandes villes, pourraient devenir si polluées. En plus, ce n'est pas seulement quelques arbres et quelques animaux qui sont atteints. Le mal provoqué par les sociétés industrielles est plus sérieux et plus profond qu'on l'avait soupçonné. Ce n'est pas seulement les réserves biologiques de la planète qui s'épuisent, car nous causons des dommages dans d'autres dimensions aussi. Plus il y a détérioration écologique, plus il y a érosion spirituelle. C'est inévitable. Trouver le repos de la balance est une tâche que beaucoup de gens doivent entreprendre, une bataille longue et acharnée.

Comme artiste, je me suis imposé la tâche de poser mon grain 
de sable du bon côté de la balance. Pour cela j'utilise les dépouilles des esprits sauvages. Je les ressucite, je les convoque, je les réunis. Nous nous lamentons sur la perte de notre lieu, nous pleurons l'agonie du paradis, le massacre de nos muses, la déroute future des enfants. Puis, le tambour change de rythme. On blasphème, on profère des imprécations, on hurle le mécontentement. Ensuite on conjure, on sacralise, on réalise.

Je crains pour le Québec. Il se croit petit mais il est un beau géant. Entouré en trois points cardinaux par une culture de vocation expansive, il ne reste que le Nord comme refuge mythique, comme réserve spirituelle. Mais tant qu'on continuera à voir le Grand Nord en termes purement économiques et politiques, les cancers de l'ambition humaine gagneront la bataille. L'identité et l'âme québécoises sont en danger, aussi bien que les indigènes de ces forêts et toundras, aussi bien que les esprits de ces lieux sauvages.

Traduit de l'espagnol par Wanda Blynn Campbell, Axel Harvey et Louise Ethier. 